International Journal of Pure and Applied Mathematics

Volume 84 No. 2 2013, 49-62

ISSN: 1311-8080 (printed version); ISSN: 1314-3395 (on-line version)

url: http://www.ijpam.eu

doi: http://dx.doi.org/10.12732/ijpam.v84i2.4

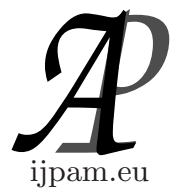

\title{
LINEAR INDEPENDENCE OF A FINITE SET OF DILATIONS BY A ONE-PARAMETER MATRIX LIE GROUP
}

\author{
David Ferrone ${ }^{1}$, Vignon Oussa ${ }^{2}$ \\ ${ }^{1}$ Department of Mathematics \\ University of Connecticut \\ Storrs-Mansfield, CT 06269, USA \\ ${ }^{2}$ Department of Mathematics \& Computer Science \\ Bridgewater State University \\ Bridgewater, MA 02325, USA
}

\begin{abstract}
Let $G=\left\{e^{t A}: t \in \mathbb{R}\right\}$ be a closed one-parameter subgroup of the general linear group of matrices of order $n$ acting on $\mathbb{R}^{n}$ by matrix-vector multiplication. We assume that all eigenvalues of $A$ are rationally related. We study conditions for which the set $\left\{f\left(e^{t_{1} A} \cdot\right), \cdots, f\left(e^{t_{m} A} \cdot\right)\right\}$ is linearly dependent in $L^{p}\left(\mathbb{R}^{n}\right)$ with $1 \leq p<\infty$.
\end{abstract}

AMS Subject Classification: 39B52

Key Words: orbit, one-parameter, groups, cross-sections, dilation equation

\section{Introduction}

In their fundamental paper [3], Edgar and Rosenblatt studied difference equations over locally compact abelian groups. They define a homogeneous linear difference equation with constant coefficients over $\mathbb{R}^{n}$ to be an equation of the type $\sum_{k=1}^{m} c_{k} f\left(x+t_{j}\right)=0$ which holds for all $x \in \mathbb{R}^{n}$ where $c_{1}, \cdots, c_{n}$ are nonzero complex scalars, $t_{1}, \cdots, t_{n}$ are distinct elements in $\mathbb{R}^{n}$, and $f$ is

Received: October 20, 2012

(c) 2013 Academic Publications, Ltd. url: www.acadpubl.eu 
a complex-valued function defined on $\mathbb{R}^{n}$. A function has linearly dependent translates when it is a solution to the difference equation. Edgar and Rosenblatt proved in $[3]$ that in $L^{p}(\mathbb{R}), 1 \leq p \leq 2$ such an equation does not have a non-trivial solution. In other words, any finite set of translates of a non-zero function is linearly independent. However, for more general Euclidean spaces, surprisingly, if $p>2 n /(n-1)$, it is possible to find non-trivial solutions for the difference equation. Very recently, during a discussion related to Edgar and Rosenblatt's paper, Speegle asked the following question. If the translations in the homogeneous linear difference equation are replaced by dilations do the same results hold? Inspired by his question, we consider equations of the type $\sum_{k=1}^{m} c_{k} f\left(M_{k} x\right)=0$, where $\left\{M_{1}, \cdots, M_{m}\right\}$ is a finite set of non-singular matrices which are elements of a one-parameter dilation group. More precisely, let $A$ be a matrix of order $n$, and let $e^{A}=\sum_{k=0}^{\infty} \frac{A^{k}}{k !}$ be an $n \times n$ non-singular matrix. Throughout the paper, we assume that all eigenvalues of $A$ contained in $i \mathbb{R}$ are rationally related. That is, if $\left\{i \beta_{1}, \cdots, i \beta_{s}\right\}$ is the set of all eigenvalues of $A$ in $i \mathbb{R}^{*}$, then $\operatorname{dim}_{\mathbb{Q}}\left\{i \beta_{1}, \cdots, i \beta_{s}\right\}=1$. Let $G=\left\{e^{t A}: t \in \mathbb{R}\right\}$ be a one-parameter analytic closed subgroup of the general linear group $G L(n, \mathbb{R})$. Thus $G$ is a matrix Lie group. Given $v \in \mathbb{R}^{n}$, the group $G$ acts on $\mathbb{R}^{n}$ as follows. $e^{t A} \cdot v=e^{t A} v$ for $v \in \mathbb{R}^{n}$. In fact, this action is just the left action given by matrix multiplications. We are interested in the following basic question. Given a matrix $A$, when is true that

$$
\sum_{k=1}^{m} c_{k} \phi\left(e^{t_{k} A} \cdot\right)=0
$$

has a non-trivial solution in $L^{p}\left(\mathbb{R}^{n}\right)$ with $1 \leq p<\infty$ ? In other words, for which function $\phi$ is the set $\left\{\phi\left(e^{t_{1} A} \cdot\right), \cdots, \phi\left(e^{t_{m} A} \cdot\right)\right\}$ linearly dependent in $L^{p}\left(\mathbb{R}^{n}\right)$ for $1 \leq p<\infty$ ? The unknowns in this equation are the vectors $\left[c_{1}, c_{2}, \cdots, c_{m}\right]$, $\left[t_{1}, t_{2}, \cdots, t_{m}\right] \in \mathbb{R}^{m}$, and the function $\phi$. We assume that $t_{1}, t_{2}, \cdots, t_{m}$ are distinct real numbers.

Let $\operatorname{supp}(\phi)$ be the support of $\phi \in L^{p}\left(\mathbb{R}^{n}\right)$. Let $K(\mathbb{R})$ be the set of all measurable functions of compact support on the real line, and let $C_{0}(\mathbb{R})$ be the set of all continuous functions vanishing at infinity. We show that there exists an open dense, co-null (with respect to the Lebesgue measure) subset of $\mathbb{R}^{n}$ which is $G$-invariant, and only contains locally compact $G$-orbits of maximal dimension. We call such subset $\Omega$. For each $v \in \operatorname{supp}(\phi) \cap \Omega$, there exists a smooth structure on the $G$-orbit of $v$ such that the map $\varphi_{v}: G v \rightarrow \mathbb{R}$ defined by $\varphi_{v}\left(e^{t A} v\right)=t$ is a diffeomorphism. Let

$$
\Lambda_{\phi}=\left\{v \in \operatorname{supp}(\phi) \cap \Omega: \phi_{v} \circ \varphi_{v}^{-1} \in C_{0}(\mathbb{R}) \text { or } L^{p}(\mathbb{R}) \text { or } K(\mathbb{R})\right\} .
$$


and let $\mathbf{m}$ be the canonical Lebesgue measure on $\mathbb{R}^{n}$. We summarize our main results in the following theorem.

Theorem 1. If $G$ is not the identity group, $A$ is traceless, and $G$ acts freely in $\Omega$ then the equation $\sum_{k=1}^{m} c_{k} \phi\left(e^{t_{k} A} \cdot\right)=0$ has no non-trivial solution in $L^{p}\left(\mathbb{R}^{n}\right)$. If there exists some $v \in \mathbb{R}^{n}$ such that the isotropy group of $v$ is isomorphic to a lattice subgroup of $\mathbb{R}$ then there is a non-trivial solution for $\sum_{k=1}^{m} c_{k} \phi\left(e^{t_{k} A} \cdot\right)=0$. Suppose that $G$ is not the identity group, and $G$ acts freely in $\Omega$. Let $\phi \in L^{p}\left(\mathbb{R}^{n}\right)-0$. If $\left\{\phi\left(e^{t_{1} A} \cdot\right), \cdots, \phi\left(e^{t_{m} A} \cdot\right)\right\}$ is linearly dependent then $\mathbf{m}\left(\Lambda_{\phi}\right)=0$ and $m>2$.

If $\phi$ is non-trivial, a direct consequence of Theorem 1 is that, if $G$ acts freely a.e. and if $\left\{\phi\left(e^{t_{1} A} \cdot\right), \cdots, \phi\left(e^{t_{m} A} \cdot\right)\right\}$ is linearly dependent then $\phi$ is not continuous.

\section{Orbital Structure and Cross-Sections}

Let us start by setting up some notation. Throughout the paper we write the identity matrix of order $n$ as $1_{n \times n}$. For the transpose of a vector $v$ we write $v^{t r}$, and all sets are assumed to be measurable. Also, it is worth to make the following remarks. First of all, the case where $p=\infty$ is not interesting. Also, suppose that Equation (1) has a non-trivial solution, and $\phi$ is a solution such that $\phi(0) \neq 0$. Then it must be case that $\sum_{k=1}^{m} c_{k}=0$. Secondly, let $\phi$ $\in L^{1}\left(\mathbb{R}^{n}\right)$ be a positive function. By integrating both sides of Equation (1), and by performing some change of variables on each term of the sum, it is easy to see that if $\phi$ is a solution of Equation (1) then $\sum_{k=1}^{m} c_{k}\left|\operatorname{det}\left(e^{-t_{k} A}\right)\right|=0$. Also, if the vector $\left[t_{1}, t_{2}, \cdots, t_{m}\right]$ and a function $\phi$ are given, then there is a rather simple procedure available to decide if $\left\{\phi\left(e^{t_{1} A} \cdot\right), \cdots, \phi\left(e^{t_{m} A} \cdot\right)\right\}$ is linearly independent.

Proposition 2. If there exists $u_{1}, u_{2}, \cdots, u_{m}$ in the domain of the function $\phi$ such that the matrix

$$
\left[\phi\left(e^{t_{i}} u_{j}\right)\right]_{1 \leq i, j \leq m}
$$

is a non-singular matrix of order $m$, then $\left\{\phi\left(e^{t_{1} A} \cdot\right), \cdots, \phi\left(e^{t_{m} A} \cdot\right)\right\}$ is linearly independent.

Proof. Suppose there exists $u_{1}, u_{2}, \cdots, u_{m}$ in the domain of the function $\phi$ such that the matrix $X=\left[\phi\left(e^{t_{i}} u_{j}\right)\right]_{1 \leq i, j \leq m}$ is non-singular, and $\phi$ satisfies Equation (1) for some nonzero vector $c=\left[c_{1}, c_{2}, \cdots, c_{m}\right]^{t r}$. We can then say 
that Equation (1) is equivalent to $X c=[0,0, \cdots, 0]^{t r}$. As a result, $c$ is a nontrivial element in the null-space of the matrix $X$. That would be a contradiction since $X$ is invertible.

If all eigenvalues of $A$ are real, we may assume that $A$ is given in real Jordan canonical form. Otherwise, we may still use a real Jordan canonical form. However, it is much more convenient to use a complexification of $\mathbb{R}^{n}$ and to use a matrix which is similar to $A$, and is of the form $D+N$, where $D$ is a diagonal matrix (with non real complex eigenvalues) and $N$ is a proper lower diagonal nilpotent matrix . We explain how such form is obtained. Let us suppose that the matrix $A$ given in real Jordan canonical form has $p$ real eigenvalues, and $q$ non real complex eigenvalues. Notice that $q$ is even. We identify $\mathbb{R}^{n}$ with $\mathbb{R}^{p} \times \mathbb{R}^{q}$ such that $n=p+q$. Next, we fix bases for $\mathbb{R}^{p} \times \mathbb{R}^{q}$ and $\mathbb{C}^{p} \times \mathbb{C}^{q}$ such that the topological embedding map

$$
\iota: \mathbb{R}^{p} \times \mathbb{R}^{q} \rightarrow \mathbb{C}^{p} \times \mathbb{C}^{q}
$$

defined by

$$
\begin{aligned}
& \iota\left(\left[v_{1}, \cdots, v_{p}, w_{1}, \cdots, w_{q}\right]^{t r}\right) \\
& =\left[v_{1}, \cdots, v_{p}, w_{1}+i w_{2}, \cdots, i w_{q}+w_{q-1}, w_{1}-i w_{2}, \cdots, i w_{q}-w_{q-1}\right]^{t r}
\end{aligned}
$$

induces the following commutative diagram:

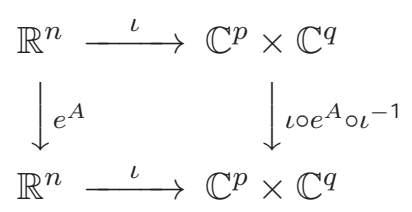

Given a matrix $A$ in real Jordan canonical form, we identify $A$ with $\iota \circ A \circ \iota^{-1}$ and $\mathbb{R}^{p} \times \mathbb{R}^{q}$ with $\iota\left(\mathbb{R}^{p} \times \mathbb{R}^{q}\right) \subset \mathbb{C}^{p} \times \mathbb{C}^{q}$. The matrix $e^{\iota \circ A \circ \iota^{-1}}$ is then given in block form with both real and complex generalized eigenvalues (see Example 8).

Definition 3. Let $\mathcal{X}$ be a $G$-invariant subset of $\mathbb{R}^{n}$. A Borel set $C \subset \mathcal{X}$ is called a cross-section for the action of $G$ in $\mathcal{X}$ if

1. $\bigcup_{t \in \mathbb{R}} e^{t A} C=\mathcal{X}$.

2. $e^{t_{1} A} C \cap e^{t_{2} A} C=\emptyset$ whenever $t_{1} \neq t_{2}$. 
Definition 4. The isotropy group of $v$ is defined as

$$
G_{v}=\left\{e^{t A} \in G: e^{t A} v=v\right\} .
$$

Definition 5. The action of $G$ on a $G$-invariant subset of $\mathbb{R}^{n}$ denoted by $\mathcal{X}$ is said to be free in $\mathcal{X}$ if for any $v$ in $\mathcal{X}, e^{t A} v=e^{s A} v$ implies $t=s$. Equivalently, if there exists a vector $v$ in $\mathcal{X}$ such that $e^{t A} v=v$ then $t=0$.

Lemma 6. If $A$ is diagonalizable with eigenvalues in $i \mathbb{R}$, there is a $G$ invariant open dense and co-null subset of $\mathbb{R}^{n}$, denoted $\Omega$ which only contains orbits of maximal dimension such that the map $v \mapsto G_{v}$ is constant on $\Omega$.

Proof. For the case where $G$ is the identity group, the proof is trivial. For the second part, suppose that $G$ is not equal to the identity group. We decompose $\mathbb{C}^{p} \times \mathbb{C}^{q}$ into direct sums of eigensubspaces such that $\mathbb{C}^{p} \times \mathbb{C}^{q}$ is identified with

$$
\mathbb{C}^{p} \oplus\left(\bigoplus_{j=1}^{q / 2} V_{j}\right) \oplus\left(\bigoplus_{j=1}^{q / 2} \overline{V_{j}}\right)
$$

and the restriction of $\iota \circ A \circ \iota^{-1}$ onto $\mathbb{C}^{p}$ is a zero matrix, the restriction of $\iota \circ A \circ \iota^{-1}$ onto $V_{k}$ is equal to a matrix $B_{k}$, and the restriction of $\iota \circ A \circ \iota^{-1}$ onto $\overline{V_{k}}$ is equal to $\bar{B}_{k}$, where $B_{k}$ is a diagonal matrix. Moreover, $\left(B_{k}\right)_{j, j}=i \beta_{k}$, and $\bar{B}_{k}$ is a diagonal matrix such that $\left(\bar{B}_{k}\right)_{j, j}=-i \beta_{k}$. Furthermore, $i \beta_{k} \neq 0$ for $1 \leq k \leq q / 2$ and $i \beta_{j} \neq i \beta_{l}$ for $j \neq l$. We recall that it is assumed that $\operatorname{dim}_{\mathbb{Q}}\left\{i \beta_{1}, \cdots, i \beta_{q / 2}\right\}=1$. Now let $\pi_{k}$ be the projection of $\mathbb{C}^{p} \times \mathbb{C}^{q}$ onto $V_{k}$. We define $\Omega=\iota^{-1}\left(\left\{v \in V:\left(\pi_{k}(v)\right)_{1} \neq 0,1 \leq k \leq q / 2\right\}\right)$. Clearly, given $v \in \Omega$, the isotropy group of $v$ is obtained by finding all $e^{t A} \in G, t \in \mathbb{R}$ such that $t \beta_{1}=2 \pi k_{1}, \cdots, t \beta_{q / 2}=2 \pi k_{q / 2}$ where $k_{1}, k_{2}, \cdots, k_{q / 2} \in \mathbb{Z}$. Finally

$$
G_{v}=\left\{e^{2 \pi r k A}: r=\operatorname{lcm}\left(\beta_{1}, \cdots \beta_{k}\right), k \in \mathbb{Z}\right\}
$$

which is a lattice subgroup of $G$.

We will show how to construct cross-sections which are different from the cross-sections given in [4]. The reason why we seek to obtain a different construction is because we would like to assert that if $G$ acts freely on at least one vector, then there is a smooth cross-section for the action of $G$ in some open, dense, co-null, and $G$-invariant subset of $\mathbb{R}^{n}$. Also, the cross-sections obtained here will allow us to make some precise computations later in the paper. The procedure used in this paper is very similar to a standard technique 
which has been extensively used by Arnal, Currey, and Dali in [1] to compute cross-sections for the coadjoint action of a class of solvable Lie groups.

Let $\iota$ be as defined in (2) and identify $\mathbb{R}^{n}$ with $\mathbb{R}^{p} \times \mathbb{R}^{q}$.

Lemma 7. Suppose that $G$ is not equal to the identity group, and that $G$ acts freely a.e. There exists a $G$-invariant, open, dense and co-null subset of $\iota\left(\mathbb{R}^{p} \times \mathbb{R}^{q}\right)$, denoted $\Omega_{\mathbb{C}}$ and a function $\mathbf{F}$ defined on $\Omega_{\mathbb{C}}$ such that the crosssection of the $G$-action in $\Omega_{\mathbb{C}}$ is

$$
\Sigma_{\mathbb{C}}=\left\{v \in \Omega_{\mathbb{C}}: \mathbf{F}(v)=0\right\}
$$

Moreover, $\Omega=\iota^{-1}\left(\Omega_{\mathbb{C}}\right)$ is a $G$-invariant open dense subset of $\mathbb{R}^{p} \times \mathbb{R}^{q}$ and a cross-section $\Sigma$ for the action of $G$ in $\Omega$ is an $(n-1)$-dimensional manifold given by $\Sigma=\iota^{-1}\left(\Sigma_{\mathbb{C}}\right)$.

Proof. Let $v \in \mathbb{R}^{n}$. Let us suppose that $\lambda_{1}, \cdots, \lambda_{n} \in \mathbb{C}$ are the diagonal entries for the matrix $\iota \circ A \circ \iota^{-1}$ which is given in the form described in (2). If the spectrum of $A$ is real, we let $V=\mathbb{R}^{n}$ and in that case the map $\iota$ is just the identity map. Otherwise, we may assume that $\mathbb{R}^{n}$ is identified with $V=\iota\left(\mathbb{R}^{p} \times \mathbb{R}^{q}\right)$ as shown in (2). Let $A=D+N$, where $D$ is a diagonal matrix with possibly complex entries, and $N$ is a proper lower triangular nilpotent matrix. Computing the action of $e^{t A}$, we have

$$
e^{t A}\left[\begin{array}{c}
v_{1} \\
v_{2} \\
\vdots \\
v_{n}
\end{array}\right]=\left[\begin{array}{c}
\xi_{1}(t, v) \\
\xi_{2}(t, v) \\
\vdots \\
\xi_{n}(t, v)
\end{array}\right]=\left[\begin{array}{c}
e^{t \lambda_{1}}\left(v_{1}+p_{1}(t, v)\right) \\
e^{t \lambda_{1}}\left(v_{2}+p_{2}(t, v)\right) \\
\vdots \\
e^{t \lambda n}\left(v_{n}+p_{n}(t, v)\right)
\end{array}\right]
$$

where for each $k, 1 \leq k \leq n$ the function $p_{k}$ is a homogeneous polynomial of degree at most $k-1$ with $p_{1}=0$. We proceed to describe how to obtain a cross-section for the action of $G$ in some open dense, $G$-invariant, co-null subset of $\mathbb{R}^{n}$ which we will describe precisely in this proof. Let

$$
\iota \circ A \circ \iota^{-1}=\left[\begin{array}{ccc}
B_{1} & & \\
& \ddots & \\
& & B_{s}
\end{array}\right], B_{k}=\left[\begin{array}{ccc}
\lambda_{B_{k}} & \\
* & \ddots & \\
& * & \lambda_{B_{k}}
\end{array}\right], \lambda_{B_{k}} \in \mathbb{C} .
$$

There are three main cases to consider. For the first case, let us assume that there is a block $B_{j}$ such that the non-zero entry shown below is located at the k-th row of matrix $\iota \circ A \circ \iota^{-1}$. Without loss of generality, we may assume that 
$j=1$.

$$
B_{1}=\left[\begin{array}{cccc}
0 & & & \\
& \ddots & & \\
& 1 & 0 & \\
& & & \ddots
\end{array}\right] \leftarrow \mathbf{k} \text {-th row } .
$$

We define $\Omega_{\mathbb{C}}=\left\{v \in V: v_{\mathbf{k}-\mathbf{1}} \neq 0\right\}$. To construct a cross-section, we solve the equation $v_{\mathbf{k}}+t v_{\mathbf{k}-1}=0$ for $t$, and we define the function $\mathbf{F}$ over $\Omega_{\mathbb{C}}$ such that $\mathbf{F}(v)=v_{\mathbf{k}}$. For the second case, we assume that case 1 does not hold, and that without loss of generality the first block $B_{1}$ has for eigenvalues $\alpha_{1}+i \beta_{1}$ where $\alpha_{1} \neq 0$. In that case, we define $\Omega_{\mathbb{C}}=\left\{v \in V: v_{1} \neq 0\right\}$. In order to construct a cross-section, we solve the equation $e^{t\left(\alpha_{1}+i \beta_{1}\right)} v_{1}=\frac{v_{1}}{\left|v_{1}\right|}$, and we set $\mathbf{F}(v)=\left|v_{1}\right|-1$. For the last case, we assume that case 2 does not hold. In other words, the spectrum of $A$ is contained in $i \mathbb{R}^{*}$ and $A$ is not diagonalizable. Let $\beta_{1} \in i \mathbb{R}^{*}$. Without loss of generality we assume that

$$
B_{1}=\left[\begin{array}{cccccc}
\beta_{1} & & & & & \\
0 & \ddots & & & & \\
& \ddots & \beta_{1} & & \\
& & 1 & \beta_{1} & \\
& & & * & \ddots & \\
& & & & \ddots & \beta_{1}
\end{array}\right] \leftarrow \text { k-th row }
$$

Let us also assume that the non-zero entry is located at the $\mathbf{k}$-th row of $\iota$ ० $A \circ \iota^{-1}$ as shown above. To construct a cross-section, we solve the equation $\operatorname{Re}\left(\left(v_{\mathbf{k}}+t v_{\mathbf{k}-1}\right) \overline{v_{\mathbf{k}-1}}\right)=0$ for $t$ and we obtain $t=-\frac{\operatorname{Re}\left(v_{\mathbf{k}} \overline{v_{\mathbf{k}}-1}\right)}{\left|v_{\mathbf{k}-1}\right|^{2}}$. Now, let $t_{v}$ be the unique solution to each of the equation solved in either case 1 , case 2 or case 3. The cross-section mapping $\sigma: \Omega_{\mathbb{C}} \rightarrow \Sigma_{\mathbb{C}}$, is defined as $\sigma(v)=e^{\left(t_{v} A\right)} v$. For the third case, $\Omega_{\mathbb{C}}=\left\{v \in V: v_{\mathbf{k}-1} \neq 0\right\}$, and $\mathbf{F}(v)=\operatorname{Re}\left(v_{\mathbf{k}} \overline{v_{\mathbf{k}-1}}\right)$. Finally, the cross-section for the action of $G$ in $\Omega_{\mathbb{C}}$ is given by

$$
\Sigma_{\mathbb{C}}=\left\{v \in \Omega_{\mathbb{C}}: \mathbf{F}(v)=0\right\} .
$$

For the first case,

$$
\Sigma_{\mathbb{C}}=\left\{\left[v_{1}, \cdots, v_{\mathbf{k}-1}, 0, v_{\mathbf{k}+1}, \cdots, v_{n}\right]^{t r} \in V: v_{\mathbf{k}-1} \neq 0\right\} .
$$

For the second case, $\Sigma_{\mathbb{C}}=\left\{\left[v_{1}, \cdots, v_{n}\right]^{t r} \in V: v_{1} \neq 0,\left|v_{1}\right|=1\right\}$. Finally, for the third case,

$$
\Sigma_{\mathbb{C}}=\left\{\left[v_{1}, \cdots, v_{n}\right]^{t r} \in V: v_{\mathbf{k}-1} \neq 0, \operatorname{Re}\left(v_{\mathbf{k}} \overline{v_{\mathbf{k}-1}}\right)=0\right\} .
$$


To complete the proof we define $\Omega=\iota^{-1}\left(\Omega_{\mathbb{C}}\right), \Sigma=\iota^{-1}\left(\Sigma_{\mathbb{C}}\right)$.

Example 8. Let

$$
A=\left[\begin{array}{cccc}
0 & 1 & 0 & 0 \\
-1 & 0 & 0 & 0 \\
1 & 0 & 0 & 1 \\
0 & 1 & -1 & 0
\end{array}\right] \text { and } \iota \circ A \circ \iota^{-1}=\left[\begin{array}{cccc}
i & 0 & 0 & 0 \\
1 & i & 0 & 0 \\
0 & 0 & -i & 0 \\
0 & 0 & 1 & -i
\end{array}\right]
$$

where

$$
\iota\left(\left[v_{1}, v_{2}, v_{3}, v_{4}\right]^{t r}\right)=\left[v_{1}+i v_{2}, v_{3}+i v_{4}, v_{1}-i v_{2}, v_{3}-i v_{4}\right]^{t r} .
$$

We have $\Omega_{\mathbb{C}}=\left\{v \in \iota\left(\mathbb{R}^{4}\right): v_{1}+i v_{2} \neq 0\right\}$,

$$
\Sigma_{\mathbb{C}}=\left\{\begin{array}{c}
{\left[v_{1}+i v_{2}, v_{3}+i v_{4}, v_{1}-i v_{2}, v_{3}-i v_{4}\right]^{t r} \in \mathbb{C}^{4}:} \\
v_{1}+i v_{2} \neq 0, v_{1} v_{3}+v_{2} v_{4}=0
\end{array}\right\},
$$

and $\Sigma=\iota^{-1}\left(\Sigma_{\mathbb{C}}\right)$.

Lemma 9. Let $v \in \Omega$. A $G$-orbit of $v$ is diffeomorphic to the real line, or the circle or is equal to $v$.

Proof. Given $A$ in the form described above. $A=D+N$ where $D$ is a diagonal matrix and $N$ is a nilpotent matrix. Thus, $e^{t A}=e^{t D} e^{t N}$. If $N \neq 0$ then $G_{v}=\left\{1_{n \times n}\right\}$. Now suppose that $N=0$ and $D \neq 0$. If there is an eigenvalue of $A$ which has a non-trivial real part, then $G_{v}=\left\{1_{n \times n}\right\}$. Otherwise, if all eigenvalues are purely imaginary, and rationally related then $G_{v}$ is a lattice subgroup of $G$ (see 6 ). Thus $G_{v}=e^{r \mathbb{Z} A}$, with $r \neq 0$. Finally, if both $N$ and $D$ are equal to zero then $G_{v}=\mathbb{R}$. There is a unique smooth structure on the $G$-orbit of $v$, and with this structure, the orbit $G v$ is endowed with a transitive smooth action by $G$. Furthermore the map $d: G / G_{v} \rightarrow G v$, with $d\left(e^{t A} G_{v}\right)=e^{t A} v$ is an equivariant diffeomorphism in the sense that $d$ is a diffeomorphism, and $d\left(e^{s A} e^{t A} G_{v}\right)=e^{s A} d\left(e^{t A} G_{v}\right)$. In fact, $G v$ is a homogenous $G$-manifold. If $G_{v}=\left\{1_{n \times n}\right\}$ then $G v$ is diffeomorphic to the real line $\mathbb{R}$. If $G_{v}=G$ then $G v$ is diffeomorphic to $\{v\}$ and if $G_{v}$ is a lattice subgroup of $\mathbb{R}$ then $G v$ is diffeomorphic to a circle. This completes the proof.

We will also need the following facts.

Lemma 10. Let $\phi: \mathbb{R} \rightarrow \mathbb{C}$ be a measurable function. If $\phi$ is a nonzero function which vanishes outside of a compact set, then $\phi$ has linearly independent translates. If $\phi \in C_{0}(\mathbb{R})$, or $L^{p}(\mathbb{R})$ for $p \in[1, \infty)$ then $\phi$ has linearly independent translates. 
Proof. The proof is in [3], cor 2.11 and Theorem 1.4.

\section{Results}

Throughout the remainder of the paper, we may assume that $G$ is not the identity group. In fact if $G$ is the identity group, any function in $L^{p}\left(\mathbb{R}^{n}\right)$ is obviously a solution to Equation (1). Let $\mathbf{m}$ be the canonical Lebesgue measure on $\mathbb{R}^{n}$. Let $\phi \in L^{p}\left(\mathbb{R}^{n}\right)$ with $1 \leq p<\infty$ be a measurable non-zero function. Let $\operatorname{supp}(\phi)$ be the support of $\phi$. Then it is clear that $\mathbf{m}(\operatorname{supp}(\phi) \cap \Omega)>0$.

Lemma 11. Suppose that $G \neq\left\{1_{n \times n}\right\}$. Fix $\Omega \subset \mathbb{R}^{n}$. If there exists $v \in \Omega$, such that $G_{v}$ is non-trivial then the equation

$$
\sum_{k=1}^{m} c_{k} f\left(e^{t_{k} A} v\right)=0
$$

has a non-trivial solution.

Proof. The proof is fairly easy. If there exists some vector $u \in \Omega$ such that $G_{u}$ is non-trivial, then it must be the case that $G_{v}$ is non-trivial for every $v \in \Omega$. Since $G$ is not equal to the identity group, the $G$-orbit of $v$ is diffeomorphic to a circle. Let us consider the unit disk $D_{n}=\{v \in \Omega:\|v\| \leq 1\}$. It is clear that for any $t \in \mathbb{R}, e^{t A}\left(D_{n}\right) \subseteq D_{n}$. Thus, letting $\phi$ be the indicator function of the unit disk, it is easy to see that $\phi(v)-\phi\left(e^{A} v\right)=0$.

Fix $\Omega \subset \mathbb{R}^{n}$. From now on, we assume that the action of $G$ is free in $\Omega$. In other words $G_{v}=\left\{I_{n \times n}\right\}$ for all $v \in \Omega$. Fix $v \in \operatorname{supp}(\phi) \cap \Omega$. We define the map $\varphi_{v}: G v \rightarrow \mathbb{R}$ with $\varphi_{v}\left(e^{t A} v\right)=t$. We observe that $\varphi_{v}$ is an analytic diffeomorphism. Given any measurable function $\beta: G v \rightarrow \mathbb{C}$, the function $\beta \circ \varphi_{v}^{-1}$ is a well-defined function of the real line which we identify with $\beta$ via the diffeomorphism $\varphi_{v}$. Moreover, notice that $\varphi_{v} \circ e^{t A} \circ \varphi_{v}^{-1}=L_{t}$ and

$$
\varphi_{v} \circ e^{t A} \circ \varphi_{v}^{-1}=L_{t} \Leftrightarrow e^{t A} \circ \varphi_{v}^{-1}=\varphi_{v}^{-1} \circ L_{t}
$$

with $L_{t}(s)=s+t$. The action of $\varphi_{v} \circ e^{t A} \circ \varphi_{v}^{-1}$ is therefore a translation action along the $G$-orbit of $v$. Now, let us suppose that there is a non-trivial solution in $L^{p}\left(\mathbb{R}^{n}\right)$ with $1 \leq p<\infty$ for equation 1 . Let $\phi$ be a non-trivial solution and $\phi_{v}$ the restriction of the function $\phi$ onto the $G$-orbit of $v \in \Omega$. Then it is clear that $\sum_{k=1}^{m} c_{k} \phi_{v}\left(e^{t_{k} A} \cdot\right)=0$ for some non-trivial $c_{k}$. 
Proposition 12. Suppose that $G \neq\left\{1_{n \times n}\right\}$. If there exists $v_{0} \in \mathbb{R}^{n}$ such that $G_{v_{0}}$ is trivial, then $c_{1} \phi\left(e^{t_{1} A} \cdot\right)+c_{2} \phi\left(e^{t_{2} A} \cdot\right)=0$ has no non-trivial solution in $L^{p}\left(\mathbb{R}^{n}\right)$ for $c_{1}, c_{2} \in \mathbb{C}$ and $t_{1}, t_{2} \in \mathbb{R}$.

Proof. First, notice that since the isotropy group of $v_{0}$ is equal to the identity group, the matrix $A$ has at least one non-zero eigenvalue which is not an element of $i \mathbb{R}^{*}$, or $A=D+N$ where $D$ is a diagonal matrix with eigenvalues in $i \mathbb{R}$ and $N$ is a non-zero lower triangular nilpotent matrix. Next, if $c_{1} \phi\left(e^{t_{1} A} v\right)+c_{2} \phi\left(e^{t_{2} A} v\right)=0$ then $\phi(v)=-\frac{c_{2}}{c_{1}} \phi\left(e^{\left(t_{2}-t_{1}\right) A} v\right)$. Without loss of generality, in order to prove the proposition, it suffices to show that equation $\phi(v)=c \phi\left(e^{t A} v\right)$ only has a trivial solution. By computing the $p$-norm of $\phi$, is it easy to see that $c=\left|\operatorname{det}\left(e^{t A}\right)\right|^{1 / p}$. Also, letting $M=e^{t A}$ for any integer $k$, if $\phi(v)=|\operatorname{det}(M)|^{1 / p} \phi(M v)$ then $\phi(v)=|\operatorname{det}(M)|^{k / p} \phi\left(M^{k} v\right)$. Now, we consider the singly generated group $\Gamma=\left\{M^{k}: k \in \mathbb{Z}\right\}$ which also acts on $\Omega$. Since $A$ has at least one eigenvalue which is not purely imaginary, then $\Gamma$ is discrete, and is a closed subgroup of $G L(n, \mathbb{R})$. It is not too hard to see that given $\gamma \in \Gamma$, if $\gamma \neq 1$, then for each point $v \in \Omega$, there exists an open subset $U \subset \Omega$ such that $\gamma U \cap U$ is empty. By Corollary 2.9.12 in [5], $\Omega / \Gamma$ has an analytic structure such that the mapping $\gamma \mapsto \gamma v$ is a submersion. We refer the reader to [4], where the authors construct a cross-section for the action of $\Gamma$. Let $\Sigma^{\circ}$ be a measurable cross-section of the action of $\Gamma$ in $\Omega$, and $\chi_{\Sigma^{\circ}}$ the characteristic function of the set $\Sigma^{\circ}$. For any $k \in \mathbb{Z}$, we obtain

$$
\left\|\phi \chi_{\Sigma^{\circ}}\right\|_{L^{p}\left(\mathbb{R}^{n}\right)}^{p}=\int_{\Sigma^{\circ}}|\operatorname{det} M|^{k}\left|\phi\left(M^{k} v\right)\right|^{p} d v=\int_{M^{-k}\left(\Sigma^{\circ}\right)}|\phi(v)|^{p} d v .
$$

However, $\|\phi\|_{L^{p}\left(\mathbb{R}^{n}\right)}^{p}$ is equal to $\sum_{k \in \mathbb{Z}}\left\|\phi \chi_{M^{-k}\left(\Sigma^{\circ}\right)}\right\|_{L^{p}\left(\mathbb{R}^{n}\right)}^{p}$, and the latter is also equal to $\sum_{k \in \mathbb{Z}}\left\|\phi \chi_{\Sigma^{\circ}}\right\|_{L^{p}\left(\mathbb{R}^{n}\right)}^{p}$. If $\left\|\phi \chi_{\Sigma^{\circ}}\right\|_{L^{p}\left(\mathbb{R}^{n}\right)}^{p}=0$ then $\phi=0$. Otherwise, $\phi$ is not an element of $L^{p}\left(\mathbb{R}^{n}\right)$. This completes the proof.

From now on, we make the following assumptions. First, for an equation of the type $\sum_{k=1}^{m} c_{k} \phi\left(e^{t_{k} A} \cdot\right)=0$, we will assume that $m>2$. Secondly, we assume that $G$ acts freely on every $G$-orbit in $\Omega$.

Lemma 13. Suppose that $G \neq\left\{1_{n \times n}\right\}$. For any $v \in \operatorname{supp}(\phi) \cap \Omega$,

$$
\sum_{k=1}^{m} c_{k} \phi_{v}\left(e^{t_{k} A} \cdot\right)=\sum_{k=1}^{m} c_{k} T_{t_{k}}\left(\phi_{v} \circ \varphi_{v}^{-1}\right)(\cdot)
$$

where $T_{t_{k}}: L^{p}(\mathbb{R}) \rightarrow L^{p}(\mathbb{R})$ such that $T_{t_{k}} f(x)=f\left(x+t_{k}\right)$. 
Proof. Let $w \in G v$, using (4), there exists a unique $t_{w} \in \mathbb{R}$ such that $w=e^{t_{w} A} v$. Thus,

$$
\sum_{k=1}^{m} c_{k} \phi_{v}\left(e^{t_{k} A} w\right)=\sum_{k=1}^{m} c_{k} \phi_{v}\left(e^{t_{k} A} e^{t_{w} A} v\right)=\sum_{k=1}^{m} c_{k} \phi_{v}\left(e^{t_{k} A} \varphi_{v}^{-1}\left(t_{w}\right)\right) .
$$

Finally,

$$
\sum_{k=1}^{m} c_{k} \phi_{v}\left(e^{t_{k} A} w\right)=\sum_{k=1}^{m} c_{k} \phi_{v}\left(\varphi_{v}^{-1}\left(t_{w}+t_{k}\right)\right)=\sum_{k=1}^{m} c_{k} T_{t_{k}} \phi_{v}\left(\varphi_{v}^{-1}\left(t_{w}\right)\right) .
$$

We recall that

$$
\Lambda_{\phi}=\left\{\begin{array}{c}
v \in \operatorname{supp}(\phi) \cap \Omega: \phi_{v} \circ \varphi_{v}^{-1} \in C_{0}(\mathbb{R}) \\
\text { or } L^{p}(\mathbb{R}) \text { or } K(\mathbb{R})
\end{array}\right\} .
$$

Proposition 14. Suppose that $G \neq\left\{1_{n \times n}\right\}$, and that $G$ acts freely in $\Omega \subset \mathbb{R}^{n}$. If $\mathbf{m}\left(\Lambda_{\phi}\right)>0$ for some $p \in[1, \infty)$ then the equation

$$
\sum_{k=1}^{m} c_{k} \phi\left(e^{t_{k} A} \cdot\right)=0
$$

does not have a non-trivial solution in $L^{p}\left(\mathbb{R}^{n}\right)$.

Proof. Let us suppose that Equation (6) has a non-trivial solution. For each $v$ in $\Lambda_{\phi}$, applying the results in Lemma 13 then the restriction of a function $\phi \in$ $L^{p}\left(\mathbb{R}^{n}\right)$ (which is a solution of the equation) to the $G$-orbit of $v_{0} \in \operatorname{supp}(\phi) \cap \Omega$ yields to the following equation.

$$
\sum_{k=1}^{m} c_{k} \phi_{v_{0}}\left(e^{t_{k} A} \cdot\right)=\sum_{k=1}^{m} c_{k} T_{t_{k}}\left(\phi_{v_{0}} \circ \varphi_{v_{0}}^{-1}\right)(\cdot)=0 .
$$

Let us suppose that $\mathbf{m}\left(\Lambda_{\phi}\right)>0$ for some $p \in[1, \infty)$ and equation (6) has a non-trivial solution in $L^{p}\left(\mathbb{R}^{n}\right)$. Using Cor 2.11 and Theorem 1.4 in [3] then $\phi_{v}\left(\varphi_{v}^{-1}(t)\right)=0$ for all $t \in \mathbb{R}$ and for all $v$ in $\Lambda_{\phi}$. Let $\chi_{\Lambda_{\phi}}$ be the characteristic function of the set $\Lambda_{\phi}$. Thus $\phi \chi_{\Lambda_{\phi}}$ is a zero function. That would be a contradiction, since $\Lambda_{\phi} \subset \operatorname{supp}(\phi)$.

Let $C\left(\mathbb{R}^{n}\right)$ be the set of all continuous function on $\mathbb{R}^{n}$. 
Corollary 15. The equation $\sum_{k=1}^{m} c_{k} \phi\left(e^{t_{k} A} \cdot\right)=0$ has no non-zero solution in $L^{p}\left(\mathbb{R}^{n}\right) \cap C\left(\mathbb{R}^{n}\right)$.

Proof. Suppose by contradiction that $\phi \in L^{p}\left(\mathbb{R}^{n}\right) \cap C\left(\mathbb{R}^{n}\right)$ is a non-trivial solution to the equation. Clearly $\mathbf{m}\left(\Lambda_{\phi}\right)>0$. According to Proposition 14 that would be a contradiction.

Now, we consider the diffeomorphism $\xi: \mathbb{R} \times \Sigma \rightarrow \Omega$ defined by $\xi(t, w)=$ $e^{t A} w$. Let $\phi$ be a function in $L^{p}\left(\mathbb{R}^{n}\right)$, and let $J_{\xi}(t, w)$ be the Jacobian of $\xi$ at $(t, w) \in \mathbb{R} \times \Sigma$. Let $L(\mathbb{R} \times \Sigma)$ be set of all Lebesgue measurable functions on $\mathbb{R} \times \Sigma$. We define the operator $\mathbf{D}: L^{p}\left(\mathbb{R}^{n}\right) \rightarrow L(\mathbb{R} \times \Sigma)$ such that

$$
\mathbf{D} \phi(w, t)=\phi(\xi(t, w))\left|\operatorname{det}\left(J_{\xi}(t, w)\right)\right|^{1 / p} .
$$

We recall the definitions of the objects $\Sigma_{\mathbb{C}}$ and $\Omega_{\mathbb{C}}$ as given in Lemma 7 . Define $\xi_{\mathbb{C}}: \mathbb{R} \times \Sigma_{\mathbb{C}} \rightarrow \Omega_{\mathbb{C}}$ such that $\xi_{\mathbb{C}}(t, w)=e^{t A} w$. Let $J_{\xi_{\mathbb{C}}}$ be the Jacobian of the $\operatorname{map} \xi_{\mathbb{C}}$.

Lemma 16. $\left|\operatorname{det}\left(J_{\xi_{\mathbb{C}}}(t, w)\right)\right|=\left|\operatorname{det}\left(e^{t A}\right)\right| \mathbf{f}_{\mathbb{C}}(w)$, where $\mathbf{f}_{\mathbb{C}}$ is some positive function defined on the cross-section $\Sigma_{\mathbb{C}}$.

Proof. Let $\lambda_{1}, \cdots, \lambda_{n} \in \mathbb{C}$ be the eigenvalues of the matrix $A$. Following the proof of Lemma 7 , we have three separate cases to consider. With some elementary computations, we obtain for case $1,\left|\operatorname{det}\left(J_{\xi_{\mathbb{C}}}(t, w)\right)\right|=\left|\operatorname{det}\left(e^{t A}\right)\right|\left|v_{\mathbf{k}-1}\right|$. For case 2,

$$
J_{\xi_{\mathbb{C}}}(t, w)=\left[\begin{array}{cccc}
\lambda_{1} e^{t \lambda_{1}} x & & & \\
* & e^{t \lambda_{2}} & & \\
\vdots & * & \ddots & \\
* & \cdots & * & e^{t \lambda_{n}}
\end{array}\right],
$$

$\left|\operatorname{det}\left(J_{\xi_{\mathbb{C}}}(t, w)\right)\right|=\left|\operatorname{det}\left(e^{t A}\right)\right|\left|\lambda_{1}\right|$, and finally for case 3 we obtain that

$$
\left|\operatorname{det}\left(J_{\xi_{\mathbb{C}}}(t, w)\right)\right|=\left|\operatorname{det}\left(e^{t A}\right)\right|\left|v_{\mathbf{k}-1}+i v_{\mathbf{k}}\right|,
$$

where $\operatorname{Re}\left(v_{\mathbf{k}} \overline{v_{\mathbf{k}-1}}\right)=0$.

Applying Lemma 16, and Lemma 12.2.2 in [2], there exists a positive function $\mathbf{f}$ defined on the cross-section $\Sigma$ such that

$$
\left|\operatorname{det}\left(J_{\xi}(t, w)\right)\right|=\left|\operatorname{det}\left(e^{t A}\right)\right| \mathbf{f}(w) \text {. }
$$

Lemma 17. Suppose that $G \neq\left\{1_{n \times n}\right\}$, and that $G$ acts freely in $\Omega \subset \mathbb{R}^{n}$. If $\phi \in L^{p}\left(\mathbb{R}^{n}\right)$ then $\|\phi\|_{L^{p}\left(\mathbb{R}^{n}\right)}=\|\mathbf{D} \phi\|_{L^{p}(\mathbb{R} \times \Sigma)}$ 
Proof. Clearly, we have that $\|\phi\|_{L^{p}\left(\mathbb{R}^{n}\right)}^{p}=\int_{\Omega}|\phi(u)|^{p} d u$ and

$$
\begin{aligned}
\|\phi\|_{L^{p}\left(\mathbb{R}^{n}\right)}^{p} & =\int_{\Sigma} \int_{\mathbb{R}}|\phi(\xi(t, w))|^{p}\left|\operatorname{det}\left(J_{\xi}(t, w)\right)\right| d t d w \\
& =\left.\left.\int_{\Sigma} \int_{\mathbb{R}}|\phi(\xi(t, w))| \operatorname{det}\left(J_{\xi}(t, w)\right)\right|^{1 / p}\right|^{p} d t d w \\
& =\int_{\Sigma} \int_{\mathbb{R}}|\mathbf{D} \phi(w, t)|^{p} d t d w \\
& =\|\mathbf{D} \phi\|_{L^{p}(\mathbb{R} \times \Sigma)} .
\end{aligned}
$$

Lemma 18. Assume that $G \neq\left\{1_{n \times n}\right\}$, that $G$ acts freely in $\Omega \subset \mathbb{R}^{n}$, and $\operatorname{det}\left(e^{A}\right)=1$. If $\phi \in L^{p}\left(\mathbb{R}^{n}\right)$ then $\mathbf{m}\left(\Lambda_{\phi}\right)>0$.

Proof. If $\operatorname{det} e^{A}=1$ then $t \mapsto \operatorname{det} e^{t A}$ is constant on $\mathbb{R}$. Now suppose by contradiction that $\phi \in L^{p}\left(\mathbb{R}^{n}\right)$ and $\mathbf{m}\left(\Lambda_{\phi}\right)=0$. Let $\mu$ be the Lebesgue measure on $\Sigma$. Let $\Sigma_{\phi}=\Sigma \cap \Lambda_{\phi}$. We observe that if $\mathbf{m}\left(\Lambda_{\phi}\right)=0$ then $\mu\left(\Sigma_{\phi}\right)=0$. Applying Lemma 17, and the fact that $\phi(\xi(t, w))=\phi_{w}\left(\varphi_{w}^{-1}(t)\right)$,

$$
\begin{aligned}
\|\phi\|_{L^{p}\left(\mathbb{R}^{n}\right)}^{p} & =\int_{\Sigma} \int_{\mathbb{R}}|\phi(\xi(t, w))|^{p}|\mathbf{f}(w)| d t d w \\
& =\int_{\Sigma}|\mathbf{f}(w)|\left(\left\|\phi_{w} \circ \varphi_{w}^{-1}\right\|_{L^{p}(\mathbb{R})}^{p}\right) d w .
\end{aligned}
$$

However,

$$
\begin{aligned}
\|\phi\|_{L^{p}\left(\mathbb{R}^{n}\right)}^{p} & =\left(\int_{\Sigma_{\phi}}|\mathbf{f}(w)|\left(\left\|\phi_{w} \circ \varphi_{w}^{-1}\right\|_{L^{p}(\mathbb{R})}^{p}\right) d w\right)+ \\
& \left(\int_{\Sigma-\Sigma_{\phi}}|\mathbf{f}(w)|\left(\left\|\phi_{w} \circ \varphi_{w}^{-1}\right\|_{L^{p}(\mathbb{R})}^{p}\right) d w\right) .
\end{aligned}
$$

Since $\Sigma_{\phi}$ is a $\mu$-null set.

$$
\|\phi\|_{L^{p}\left(\mathbb{R}^{n}\right)}^{p}=\int_{\Sigma-\Sigma_{\phi}}|\mathbf{f}(w)|\left(\left\|\phi_{w} \circ \varphi_{w}^{-1}\right\|_{L^{p}(\mathbb{R})}^{p}\right) d w .
$$

Thus $\|\phi\|_{L^{p}\left(\mathbb{R}^{n}\right)}^{p}$ is either undefined or infinite. That would be a contradiction.

Recall that $\operatorname{det}\left(e^{A}\right)=\exp (\operatorname{trace}(A))$. 
Proposition 19. Assume that $G \neq\left\{1_{n \times n}\right\}$, and that $G$ acts freely in $\Omega \subset \mathbb{R}^{n}$. If trace $(A)=0$ then equation $\sum_{k=1}^{m} c_{k} \phi\left(e^{t_{k} A} \cdot\right)=0$ has no non-trivial solution in $L^{p}\left(\mathbb{R}^{n}\right)$.

Proof. The proof follows from Lemma 18.

Example 20. Coming back to Example 8,

$$
\exp (t A)\left[\begin{array}{l}
v_{1} \\
v_{2} \\
v_{3} \\
v_{4}
\end{array}\right]=\left[\begin{array}{c}
v_{1} \cos t+v_{2} \sin t \\
v_{2} \cos t-v_{1} \sin t \\
\left(v_{3}+t v_{1}\right) \cos t+\left(t v_{2}+v_{4}\right) \sin t \\
\left(v_{4}+t v_{2}\right) \cos t-\left(v_{3}+t v_{1}\right) \sin t
\end{array}\right]
$$

Notice that $A$ is traceless. Thus equation $\sum_{k=1}^{m} c_{k} \phi\left(e^{t_{k} A} \cdot\right)=0$ has no nontrivial solution in $L^{p}\left(\mathbb{R}^{4}\right)$.

\section{References}

[1] A. Didier, C. Bradley, D. Bechir, Canonical coordinates for a class of solvable groups, Monatsh. Math., 166, No. 1 (2012), 19-55.

[2] B.A. Dubrovin, A.T. Fomenko, S.P. Novikov, Modern Geometry Methods and Applications. Part I. The Geometry of Surfaces, Transformation Groups, and Fields, Springer-Verlag, USA (1992).

[3] G. Edgar, J. Rosenblatt, Difference equations over locally compact Abelian groups, Transactions AMS, 253 (1979), 273-289.

[4] D. Larson, E. Schulz, D. Speegle, K. Taylor, Explicit cross-sections of singly generated group actions, Appl. Numer. Harmon. Anal., Harmonic Analysis and Applications (2006), 209-230.

[5] V.S. Varadarajan, Lie Groups, Lie Algebras, and their Representations, Springer-Verlag, USA (1984). 